\section{Unusual spinal tuberculosis after adequate chemotherapy for lymph node tuberculosis in an immunocompetent man}

\author{
Stephen P O'Hickey, Alan Pithie, \\ Craig Skinner
}

\begin{abstract}
A 35 year old man developed paraplegia due to an epidural mass 15 months after completion of a full chemotherapy course for pulmonary and lymph node Mycobacterium bovis infection. His cellular immune function was normal after treatment. It is suggested that the lesion was a granulomatous healing response rather than bacteriological recurrence.
\end{abstract}

(Thorax 1992;47:986-987)

Relapse of tuberculosis is rare after appropriate chemotherapy for sensitive organisms in a compliant, immunologically competent patient. We report the development of an exuberant granulomatous mass in the vertebral canal causing paraplegia 15 months after completion of chemotherapy for pulmonary and lymph node tuberculosis due to Mycobacterium bovis, which was fully sensitive to the drugs used.

\section{Case report}

A 35 year old Asian man was admitted to hospital with a two month history of fever, cough, and weight loss $(13 \mathrm{~kg})$. He smoked 10 cigarettes a day and drank up to one bottle of spirits a day. He had gross cervical lymphadenopathy. His chest radiograph showed extensive upper lobe shadowing. The Mantoux test (10 TU) reaction was negative. Sputum smears showed numerous acid fast bacilli. Standard chemotherapy was started (rifampicin, isoniazid, ethambutol, and pyrazinamide) plus prednisolone $\mathbf{4 0} \mathrm{mg}$ daily.

Twice weekly Mantoux tests were performed and conversion to positive occurred on the 16th day of treatment, at which time the dose of prednisolone was reduced. ${ }^{1}$ Prednisolone was discontinued on the 36th day of treatment and the patient went home on day 44. Throughout this time his cervical lymphadenopathy was unchanged. His subsequent progress was complicated by relapse of the cervical lymphadenopathy, which settled with continued antituberculosis chemotherapy, aspiration, and a two month course of prednisolone. Ethambutol and pyrazinamide were given for four months and rifampicin and isoniazid for nine months.

Pretreatment sputum and urine samples showed acid fast bacilli. Culture yielded $M$ bovis sensitive to isoniazid, rifampicin, ethambutol, and streptomycin. After four months' treatment the sputum, urine, and lymph node aspirates still showed acid fast bacilli, but all cultures were negative.

The cellular immune function was assessed in vitro on the basis of lymphocyte proliferation in response to mycobacterial antigens, purified protein derivative (PPD) and cytotoxic $\mathrm{T}$ cell activity. ${ }^{2}$ Initial assessment at the time of admission showed extremely poor proliferation and cytotoxic $T$ cell activity. Four months later in vitro lymphocyte proliferation was high and cultured lymphocytes showed substantial cytolytic activity against both PPD presenting and antigen nonpresenting autologous macrophages.

The patient returned 15 months after completing chemotherapy, unable to walk and with a two week history of back pain and leg weakness. There was pronounced quadriceps wasting, absence of knee and ankle jerks, and patchy loss of sensation over the legs. Lumbar spine radiographs were normal. A myelogram showed a circumferential narrowing of the dural sac from the upper border of the second lumbar vertebra to the level of the sacral cul-de-sac, suggesting an extensive extradural obstructing lesion. Spinal computed tomography confirmed extradural block by a mass in the vertebral canal extending from the second to the fourth lumbar vertebra, with no evidence of bony erosion or widening of the vertebral canal (figure). Two core biopsy specimens from the second lumbar vertebra were normal. A diagnosis of an intraspinal tumour was made and lumbar laminectomy via a posterior approach was undertaken. The dura was covered by soft grey tissue. This was removed and placed in formol saline. Histological examination showed this to be granulomatous inflammation with focal caseating necrosis. No acid fast bacilli were demonstrated by auramine rhodamine stain. Culture of the tissue was not possible. In view

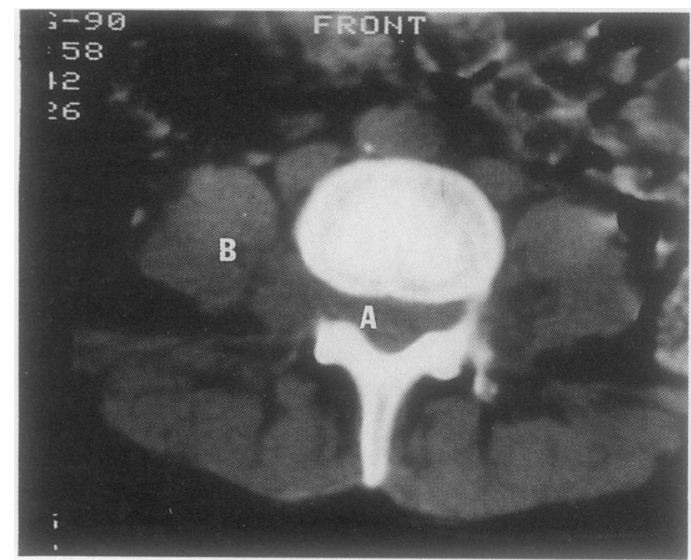

Computed tomogram of the lumbar spine showing the extradural mass $(A)$ extending from the spinal canal to the psoas muscle $(B)$. 
of the histology a further course of antituberculous chemotherapy was commenced. At that time cellular immunity, as measured in vitro in terms of lymphocyte proliferation in response to PPD, and cytolytic capacity remained normal. The patient subsequently recovered good neurological function and remains well.

\section{Discussion}

Spinal tuberculous lesions masquerading as a tumour without disease of the bone have been reported. Three were seen in a series of 190 cases of spinal tuberculosis reported by Rahman and colleagues ${ }^{3}$; all had had a preoperative diagnosis of tumour and all had responded to decompression. Babhulkar et $a l^{4}$ reported 10 in a series of 220 cases with extraosseous, extradural tuberculous disease; the lesion is described as a thick granulomatous membrane compressing the spinal cord. None of these patients had been given antituberculous chemotherapy. In our patient the primary lesion of $M$ bovis infection may have been in the intestine with lymphatic spread throughout the abdomen and to the spine.

Our patient had received an adequate course of chemotherapy for a fully sensitive organism. We believe him to have been fully compliant, though we did not check this and cannot completely exclude non-compliance as a factor. Cytotoxic T cells, which lyse both autologous mycobacteria infected macrophages and non-infected "bystander" macrophages, are known to play a part in the normal immune response to mycobacterial infection, and they may mediate delayed type hypersensitivity reactions. ${ }^{5}$ Kumararatne and colleagues $^{2}$ have postulated that recurrence of tuberculosis despite adequate chemotherapy may sometimes be related to an inability to generate cytolytic $\mathrm{T}$ cells capable of recognising mycobacterial antigens. Cytolytic activity was low initially in our patient when he was very ill and had a negative tuberculin test response. Immune paresis is well recognised in acute severe tuberculosis, occurring in up to $20 \%$ of patients with active disease. ${ }^{6}$ The paresis resolved once he had improved and had recovered his tuberculin skin reactivity, and his legs remained normal at the time of his later presentation. His initial impairment of cell mediated immunity to tuberculous antigen presumably therefore reflects immune suppression due to his severe infection and his chronic alcohol abuse rather than any constitutional immunoincompetence.

Although the spinal lesion could have been due to bacteriological reactivation, we consider this unlikely. Reinfection may cause a second episode of tuberculosis aftèr a full course of chemotherapy, ${ }^{7}$ but this seems an unlikely explanation for a granulomatous lesion presenting 15 months after completion of chemotherapy. We suggest that this clinical recurrence was due to a granulomatous healing response, which, had it occurred elsewhere (in the lung, for example), might have been of little clinical importance.

1 Onwubalili JK, Scott GM, Smith H. Acute respiratory distress related to chemotherapy of advanced pulmonary tuberculosis: a study of two cases and review of the literature. $Q J$ Med 1986;230:599-610.

2 Kumaratne DS, Pithie AS, Drysdale P, Gaston JSH, Kiessling R, Iles PB, et al. Specific lysis of mycobacterial antigen-bearing macrophages by class II MHC-restricted polyclonal $\mathrm{T}$ cell lines from healthy donors or patients with tuberculosis. Clin Exp Immunol 1990;80:314-23.

3 Rahman NM, Al-Arabi K, Khan FA. Atypical forms of spinal tuberculosis. Acta Neurochir-(Wien) 1987;88: spinal 26 . 33 .

4 Babhulkar SS, Tayade WB, Babhulkar SK. Atypical spinal tuberculosis. J Bone Joint Surg 1984;66:239-42.

5 Lowrie DB. Is macrophage death on the field of battle essential to victory, or a tactical weakness in immunity against tuberculosis? Clin Exp Immunol 1990;80:301-35.

$6 \mathrm{Nash}$ DR, Douglas JE. Anergy in active pulmonary tuberculosis: a comparison between positive and negative reactors and an evaluation of 5 TU and 250 TU skin test doses. Chest 1980;77:32-7.

7 Ormerod P, Skinner C. Reinfection tuberculosis: two cases in the family of a patient with drug-resistant disease. Thorax 1980;35:56-9. 